\title{
Optimisation of the Low-Frequency Response of a Multi-stage Bioelectric Amplifier for Electrocardiogram (ECG) Recording
}

\author{
Martin J. Burke ${ }^{1, a}$ and Kerstin Kofler ${ }^{2}$ \\ ${ }^{1}$ Dept. of Electronic and Electrical Engineering, Trinity College, University of Dublin, College Green, Dublin 2, Ireland. \\ ${ }^{2}$ Dept. of Engineering and IT, Medical Engineering, Carinthia University of Applied Sciences, 9020 Klagenfurt, Austria.
}

\begin{abstract}
This paper reports the optimisation of the low-frequency response of a multi-stage bioelectric amplifier intended for use in the measurement of the human electrocardiogram (ECG) using high-impedance un-gelled electrodes. The frequency response was optimised to meet international performance requirements for electrocardiographic recorders, in particular the International Electrotechnical Commission 60601 standards [1,2]. The pole and zero locations of a multi-stage amplifier configuration were optimised to meet both time and frequency domain specifications. The optimum assignment for a three stage amplifier, having two differential stages and a differential-to-single-ended conversion stage was established by evaluating the performance of a number of circuit configurations. The optimum configuration was found to be two differential stages with a gain of $20 \mathrm{~dB}$ each and a differential-to single-ended output stage with unity gain. The $-3 \mathrm{~dB}$ pole is placed at $0.028 \mathrm{~Hz}$ and a zero at $0.0028 \mathrm{~Hz}$ in the first and second stages to give an overall $-3 \mathrm{~dB}$ bandwidth of $0.043 \mathrm{~Hz}$. In addition, the pole of the input ac coupling network was placed at $0.0028 \mathrm{~Hz}$ in order to meet the undershoot and recovery slope requirements in the narrow pulse response.
\end{abstract}

\section{Introduction}

The low-frequency response of the bioelectric amplifier used in recording the human electrocardiogram (ECG) is of the utmost importance due to the clinical significance of such recordings. Appropriate magnitude and phase responses are needed in order to prevent distortion of the ECG signal profile and adverse changes in the waveform morphology. The nature of the distortion which can arise in the ECG signal due to poor low frequency response in the recording amplifier has been well documented for several decades and has serious diagnostic implications $[3,4]$. The type of distortion which results from poor low frequency response has a detrimental effect primarily on the T-wave, the S-T segment and the Q-T interval.

In addition to hospital oriented ECG recorders, there has been a substantial increase in recent years in the number of portable ECG recorders. Many of these are used in non-clinical scenarios such as general practice, sports medicine, physiology and even on the factory floor. This has led to an increase in battery-operated and low-power equipment with the associated changes in instrumentation design. Consequently trends have changed in the design of the electronic amplifiers for ECG recording. Lower power supply voltages have tended to make amplifier front-end stages ac coupled rather than dc coupled as in the past. This allows electrode polarisation voltages to be eliminated at the input so that they do not saturate the front-end stage of the amplifier. Recent advances in electrode technology have increased the magnitude of these polarisation voltages. Ac coupling also facilitates maintaining a high gain in the early stages of the amplifier to preserve the signal-to-noise ratio. Low-power technology has meant that the gain-bandwidth product of operational amplifiers has fallen so that the gain required in ECG amplifiers is often spread over several stages. This has led to an increase in multi-stage amplifier configurations and a consequent increase in the complexity of the associated frequency response at both the high and low ends of the spectrum. The authors have concentrated the effort in this paper on investigating the optimisation of the lowfrequency response of multi-stage bioelectric amplifiers intended for use in ECG signal recording.

\section{Background}

Standards for the performance requirements of ECG recorders have been developed in the US by the American National Standards Institute (ANSI) with recommendations made by the American Heart Association (AHA) and in Europe by the International Electrotechnical Commission (IEC). These have changed much over the decades since they were first introduced and have taken account of technological developments. Most recent standards have made an effort to merge the US and EU requirements to make them almost identical. This has, in fact, added strength to both sets of standards

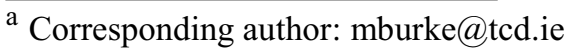


and increased the usage of the IEC 60601 standard, which is now accepted worldwide [1,2].

\subsection{Frequency domain requirements}

Early standards for ECG recorder performance were based on recommendations issued by the AHA and the IEEE in 1967 [5,6]. These standards required that the magnitude of the frequency response be within $\pm 0.5 \mathrm{~dB}$ of the mid-band gain within the frequency range $0.67 \mathrm{~Hz}-$ $150 \mathrm{~Hz}$. The low-frequency value was based on a practical lowest-heart rate value of 40 beats-per-minute (bpm). The phase response was required to be no greater than that caused by a single-pole high-pass filter having a $-3 \mathrm{~dB}$ cut-off frequency of $0.05 \mathrm{~Hz}[5,6]$. These magnitude and phase response requirements are shown in Figure 1.

More recent standards issued by both the EU and the US [1,2] require that the amplitude response of an ambulatory ECG recorder shall be within $\pm 3 \mathrm{~dB}$ of the response at $5 \mathrm{~Hz}$, within a frequency band of $0.05 \mathrm{~Hz}$ to at least $55 \mathrm{~Hz}$. A phase response requirement is not specified but the merits of a single-pole high-pass filter having a cut-off frequency of $0.05 \mathrm{~Hz}$ are still cited as example. Instead of the phase response requirement a time-domain specification has been introduced.
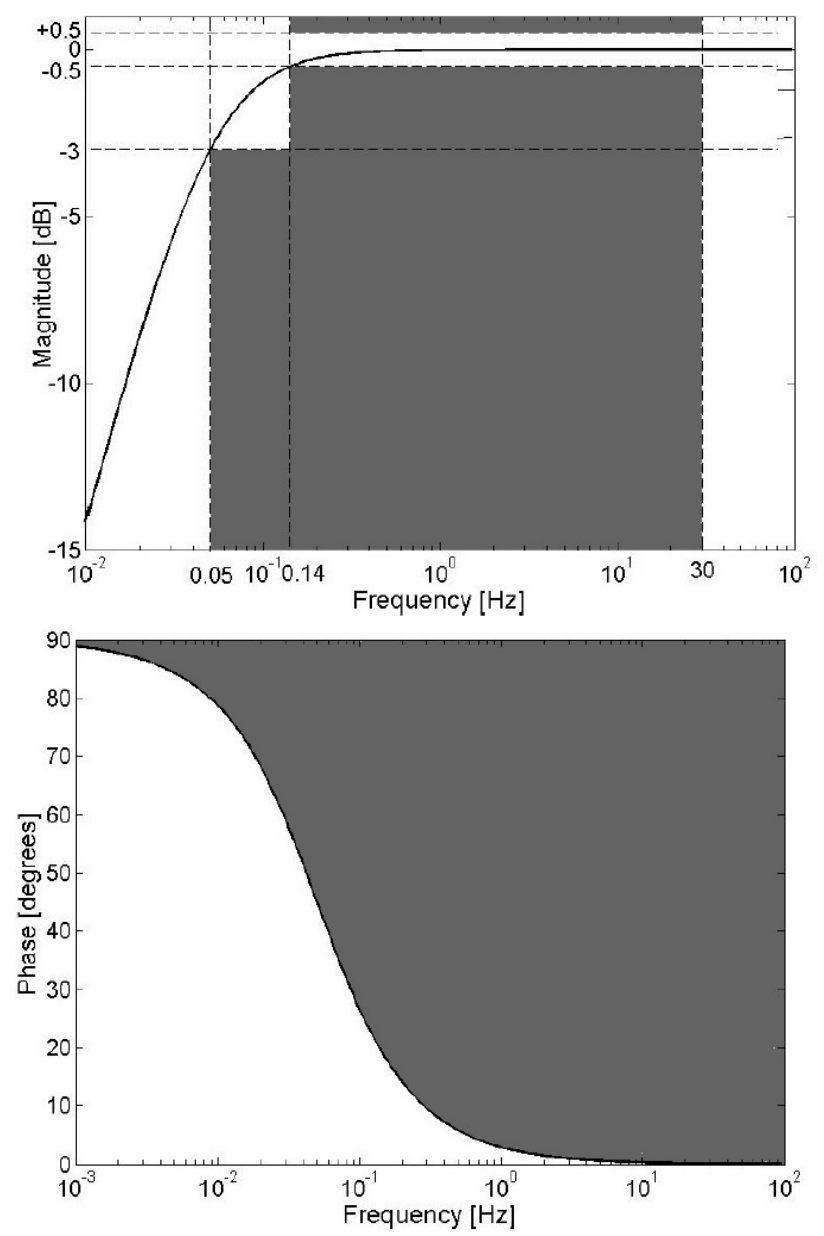

Figure 1. Magnitude and phase response requirements.

\subsection{Time domain requirements}

The IEC 60601 most recent standards, which are now closely aligned and merged with those of ANSI, have introduced two time-domain methods of testing ECG recorder performance. These are both shown in Figure 2. Method A was introduced in a previous IEC standard and allows a maximum baseline undershoot of $0.1 \mathrm{mV}$ in the transient response to a narrow rectangular pulse of $3 \mathrm{mV}$ amplitude and $100 \mathrm{~ms}$ duration with a maximum recovery slope of $0.3 \mathrm{mV} / \mathrm{s}$ between pulses, as can be seen in Figure 2. Method B, on the other hand, uses a triangular wave to model the QRS complex of the ECG. An isosceles triangular wave of peak amplitude of $1.5 \mathrm{mV}$ and a duration varying between $20 \mathrm{~ms}$ and $200 \mathrm{~ms}$ with a repetition rate of less than $1 \mathrm{~Hz}$ is applied as input to the recorder. The amplitude of the peak of the triangle must maintain a variation within $+0 \mathrm{~dB}$ and $-1 \mathrm{~dB}(-10 \%)$ as the pulse duration is varied between $20 \mathrm{~ms}$ and $200 \mathrm{~ms}$. This can be seen in Figure 2. In the studies carried out by the authors, the rectangular pulse of Method A was preferred.

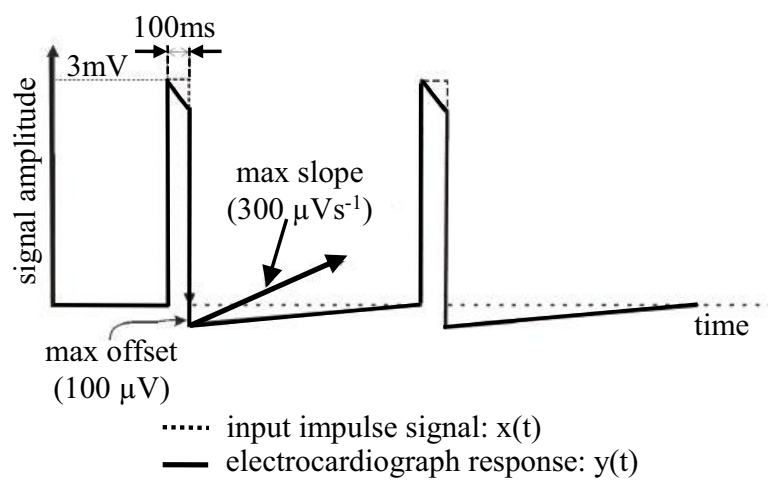

Method A: Rectangular Pulse

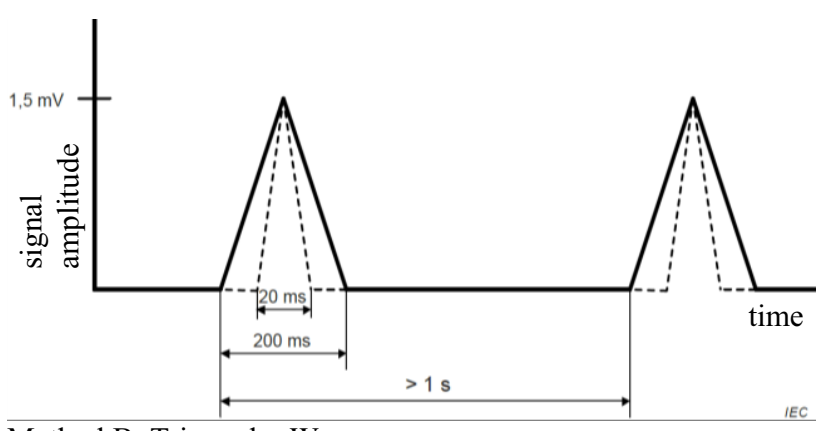

Method B: Triangular Wave

Figure 2. Transient response requirements [adapted from 1,2]

\section{Single-stage amplifiers}

\subsection{Non-inverting high-pass filter}

The first amplifier stage of interest is the simple $1^{\text {st }}$-order high-pass filter shown in Figure 3. This configuration was used as the reference standard in the early performance specifications $[5,6]$ where the $-3 \mathrm{~dB}$ cut-off frequency was $0.05 \mathrm{~Hz}$. It is still cited as a comparative reference in more recent specifications [1,2]. The steadystate transfer function of this stage is of the form:

$$
\frac{V_{o}}{V_{i}}=\frac{j \omega C_{1} R_{l}}{1+j \omega C_{1} R_{1}}
$$



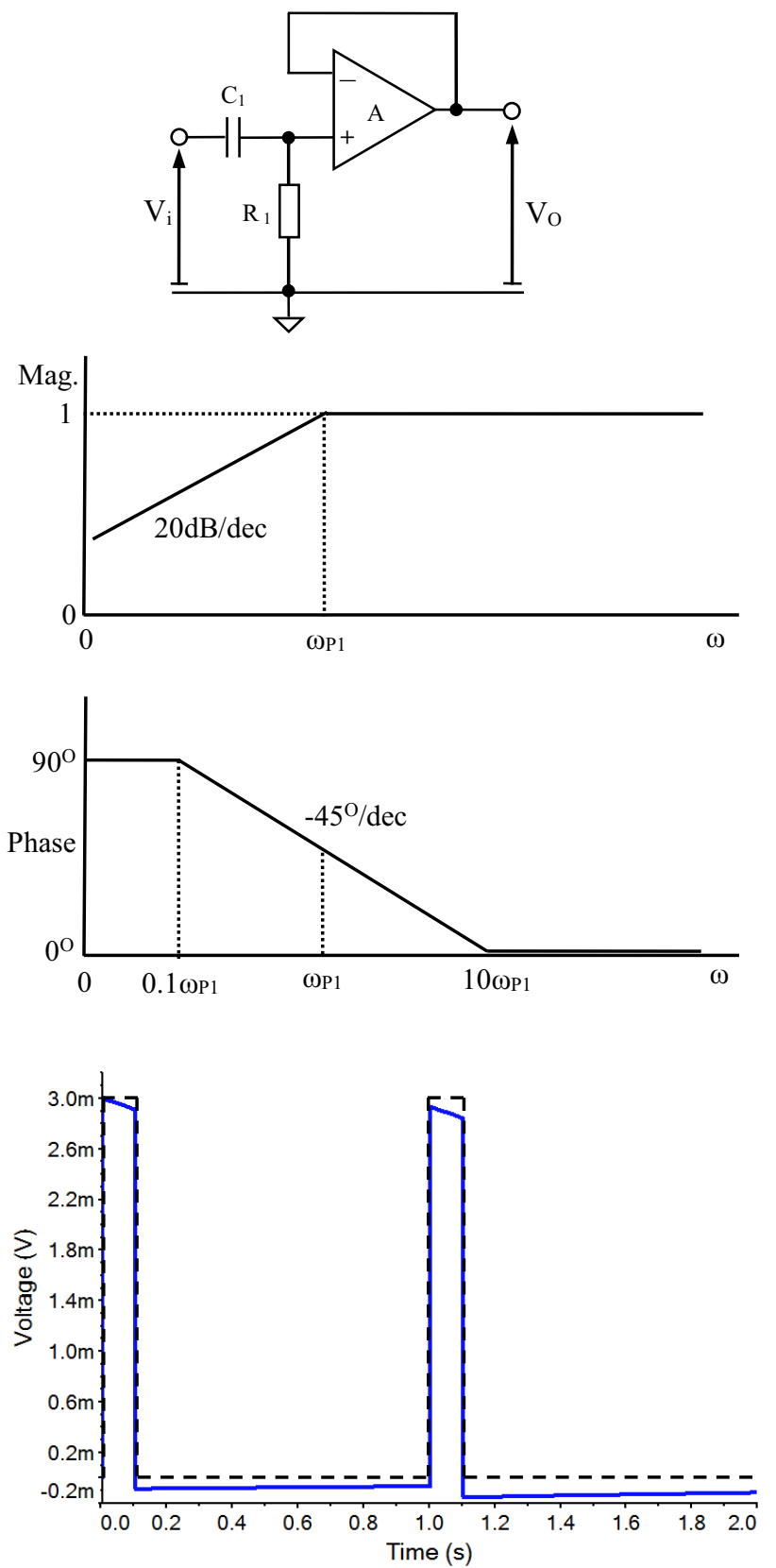

Figure 3. Magnitude, phase and pulse response of $0.05 \mathrm{~Hz} \mathrm{HPF}$.

Idealised Bode plots of the magnitude and phase of the frequency response are also shown in Figure 3. The highfrequency pass-band gain is unity. It can be seen that there is a pole at a radian frequency of $\omega_{P I}=1 /\left(C_{l} R_{l}\right)$ which corresponds to the $-3 \mathrm{~dB}$ cut-off frequency of the high-pass response. The response of this filter in the time domain to the narrow input pulse which is defined in Figure 2 can also be seen in Figure 3. The undershoot in the response is $0.093 \mathrm{mV}$ and the recovery slope is $29 \mu \mathrm{V} / \mathrm{s}$. This indicates that this filter meets all of the performance criteria for ECG amplifiers.

\subsection{Non-inverting high-pass amplifying stage}

The next stage of interest is the $1^{\text {st }}$-order high-pass amplifier stage shown in Figure 4. This stage is ac coupled but has a gain in the pass band. It has a steadystate transfer function of the form:

$$
\frac{V_{o}}{V_{i}}=\frac{\left[1+j \omega C_{1}\left(R_{1}+R_{2}\right)\right] j \omega C_{3} R_{3}}{\left(1+j \omega C_{1} R_{1}\right)\left(1+j \omega C_{3} R_{3}\right)}
$$

It can be seen that this stage has an in-band highfrequency gain of $A_{V}=\left(R_{l}+R_{2}\right) / R_{l}$. The $-3 \mathrm{~dB}$ pole in the high-pass response is again located at a radian frequency of $\omega_{P I}=1 /\left(C_{l} R_{1}\right)$. A Bode plot of the magnitude and phase of this stage is also shown in Figure 4. It can be seen that a network has been included at the input in the form of resistor $R_{3}$ and capacitor $C_{3}$ to ensure that the stage is ac coupled and that dc potentials do not appear at the input to the amplifier. If this network were omitted, the gain of this stage would level out at unity, at the zero in the frequency response which is located at a radian frequency of $\omega_{Z I}=1 /\left[C_{I}\left(R_{I}+R_{2}\right)\right]=\omega_{P I} / A_{V}$. The zero also has the effect of restoring the phase back to zero as $\omega \rightarrow 0$, as shown by the dashed lines in Figure 4 . In this case the amplifier stage could be saturated by electrode polarisation voltages at the input.

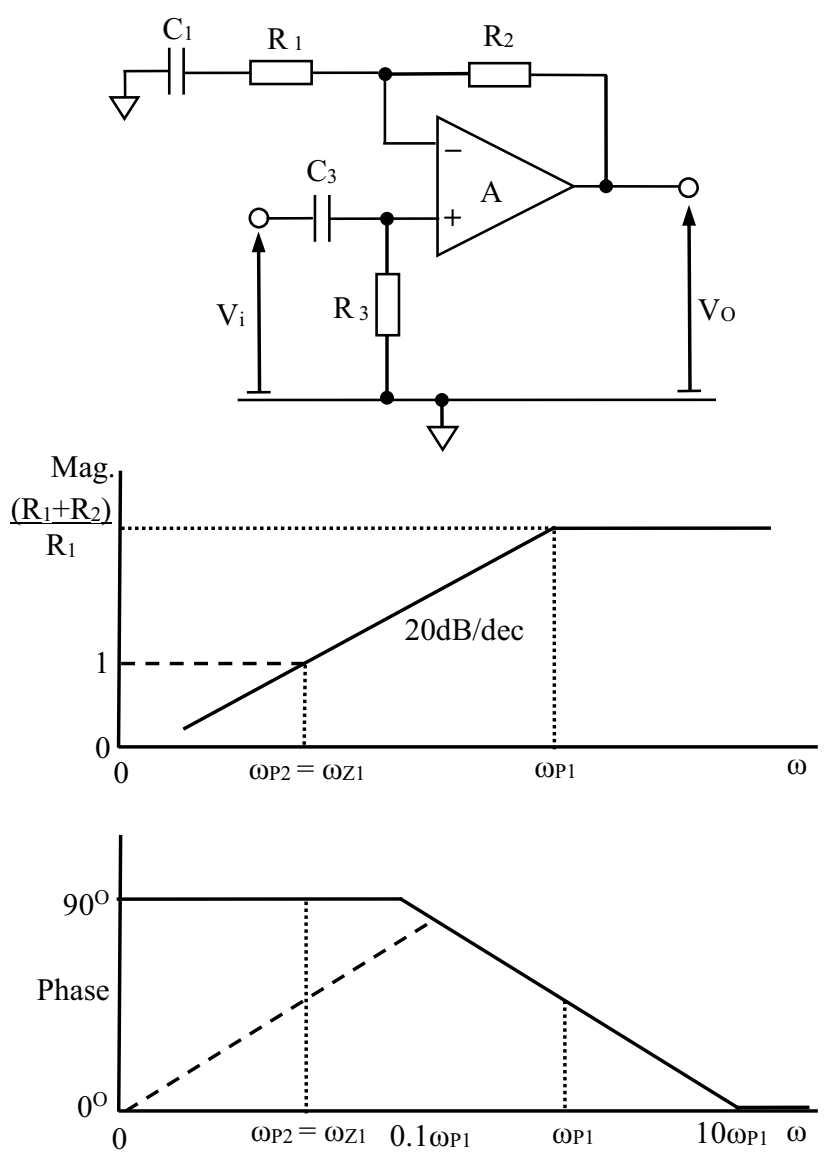

Figure 4. Magnitude and phase Bode plots of 1-stage amplifier

In order to get an overall response of the form of a highpass filter, the lower frequency pole, $\omega_{\mathrm{P} 2}$, of the input network is arranged to cancel the zero in the response of the feedback network by a choice of time constants such that $C_{3} R_{3}=C_{1}\left(R_{1}+R_{2}\right)$. In this case the transfer function of the stage becomes:

$$
\frac{V_{o}}{V_{i}}=\frac{j \omega C_{3} R_{3}}{\left(1+j \omega C_{1} R_{1}\right)}=\frac{j \omega C_{1}\left(R_{1}+R_{2}\right)}{\left(1+j \omega C_{1} R_{1}\right)}
$$

This is depicted by the solid line responses in Figure 4.

It was decided to explore whether or not the frequency response of the single high-pass stage could be 
improved by not implementing an exact cancelation of the pole and zero, but to allow a degree of mismatch in their locations. In particular the effect on the phase of varying the location of the pole on either side of the zero was of interest. Figure 5 shows plots of the magnitude and phase of a single-stage amplifier having a noninverting gain of $20 \mathrm{~dB}$.
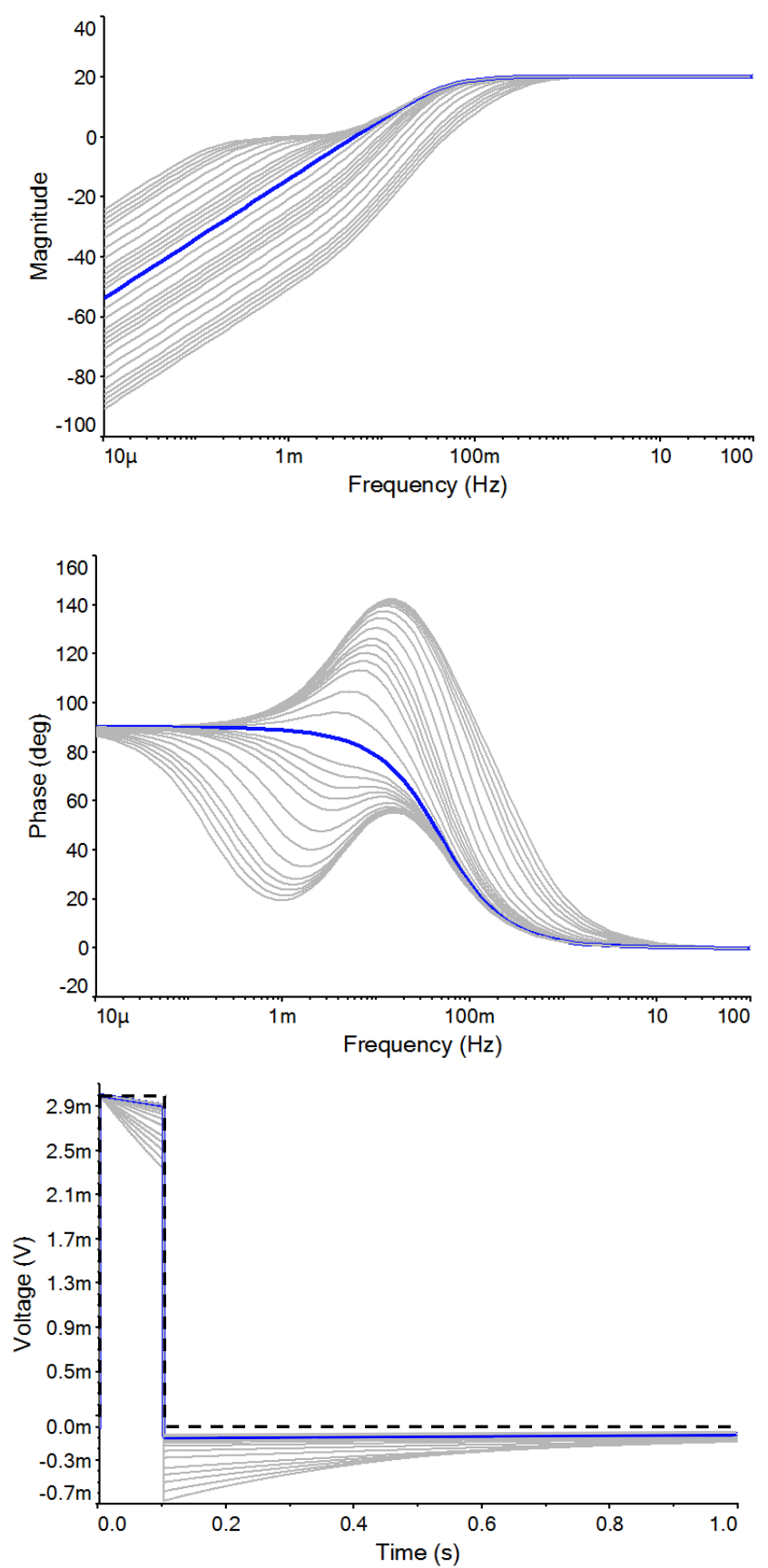

Figure 5. Magnitude, phase and pulse responses of 1-stage amp

An ideal op-amp model was used in MultiSim for circuit simulations so that its properties would not influence the results. The heavy curves in Figure 5 represent the case where $C_{3} R_{3}=C_{1}\left(R_{1}+R_{2}\right)$ with precise pole-zero cancellation. Moving the location of the pole above that of the zero increases the phase above the phase of the perfect cancellation curve, and also raises the $-3 \mathrm{~dB}$ cut-off frequency, which does not improve the response. Moving the location of the pole below that of the zero, on the other hand, lowers the phase compared with the perfect cancellation curve by a small amount at in-band frequencies and lowers the frequency at which the gain falls from $0 \mathrm{~dB}$. However, even when the frequency of the pole is lowered significantly, there is only a small reduction of the phase at in-band frequencies. The very small benefits gained in phase may be outweighed by the increased initialisation time of the amplifier. Figure 5 also shows the effect of varying the pole location on the response to the narrow $100 \mathrm{~ms}$ pulse. It can be seen that lowering the frequency of the pole location gives a slight reduction in undershoot and recovery slope of the pulse response, which are again only marginal and barely noticeable.

\section{Multi-stage amplifiers}

\subsection{Two-stage differential amplifier}

The schematic diagram of a simple differential amplifier is shown in Figure 6. This has a single cross-coupled input stage with differential input voltages $V_{1}$ and $V_{2}$. This is followed by a differential-to-single-ended conversion stage providing the output voltage, $\mathrm{V}_{\mathrm{O}}$. The $\mathrm{A}$ and $\mathrm{B}$ designation of components will nominally have the same values.

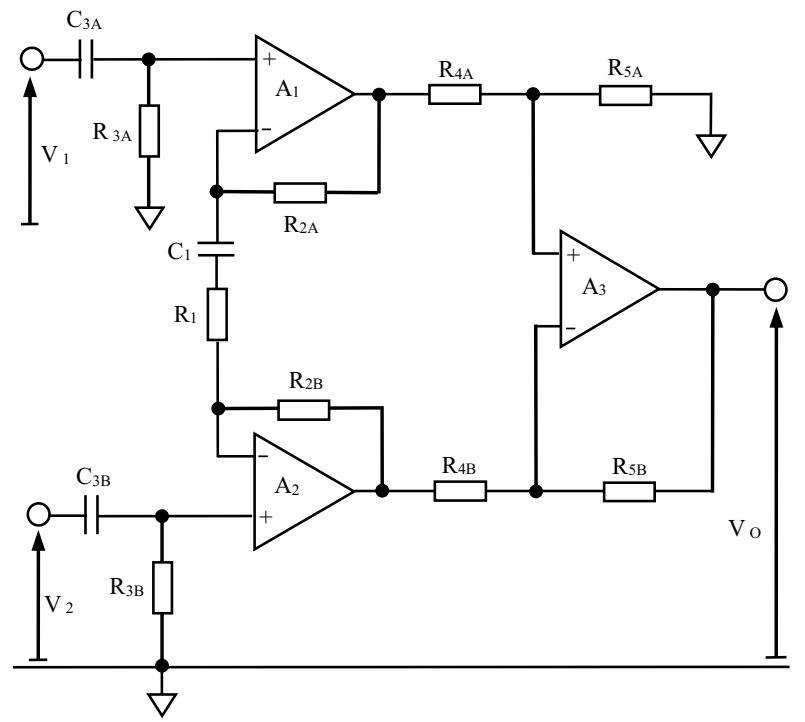

Figure 6. Schematic Diagram of a Differential Amplifier

The steady-state transfer function of this amplifier structure is given for a differential input $V_{1}-V_{2}$ as:

$$
\frac{V_{o}}{V_{1}-V_{2}}=\frac{R_{5}}{R_{4}} \frac{\left[1+j \omega C_{1}\left(R_{1}+2 R_{2}\right)\right] j \omega C_{3} R_{3}}{\left(1+j \omega C_{1} R_{1}\right)\left(1+j \omega C_{3} R_{3}\right)}
$$

Very often $\mathrm{R}_{5}=\mathrm{R}_{4}$ so that the output stage has unity gain, to maximize the CMRR. In this case the in-band differential gain of this stage is given as $A_{V}=\left(R_{l}+\right.$ $\left.2 R_{2}\right) / R_{l}$. The $-3 \mathrm{~dB}$ cut-off frequency is again located at $\omega_{P I}=1 /\left(C_{l} R_{l}\right)$ while the zero is located at $\omega_{Z I}=1 /\left[C_{l}\left(R_{I}\right.\right.$ $\left.\left.+2 R_{2}\right)\right]=\omega_{P I} / A_{V V}$. The pole due to the input network, which is identical at each input terminal, is again located at a frequency $\omega_{P 2}=1 /\left(C_{3} R_{3}\right)$. The same pole-zero cancellation as was implemented in the single stage amplifier can be obtained in the differential amplifier by 
choosing $C_{3} R_{3}=C_{1}\left(R_{1}+2 R_{2}\right)$. Plots of the magnitude and phase of the two stage differential amplifier are shown in Figure 7. Varying the location of the pole $\omega_{p 2}$ has the same effect as in the equivalent single stage amplifier. The curves therefore show only two unmatched locations for this pole, one at the geometric mean of the pole $\omega_{P I}$ and the zero $\omega_{Z 1}$ that is at a frequency $\omega=$ $\sqrt{ } \omega_{P I} \omega_{Z I}$ and the other below the zero by the same factor as the geometric mean is above it. The pulse response of Figure 8 , shows the detail at the lower end of the trailing edge of the pulse. There is little benefit to be gained from lowering the location of the pole and it has negligible effect on the undershoot and recovery slopes. Increasing the frequency of the pole location causes the undershoot to exceed $100 \mu \mathrm{V}$ and the recovery slope to exceed $300 \mu \mathrm{Vs}^{-1}$. It therefore makes most sense to apply the pole-zero cancellation principle as in the case of the single-stage amplifier and choose $C_{3} R_{3}=C_{1}\left(R_{1}+2 R_{2}\right)$.
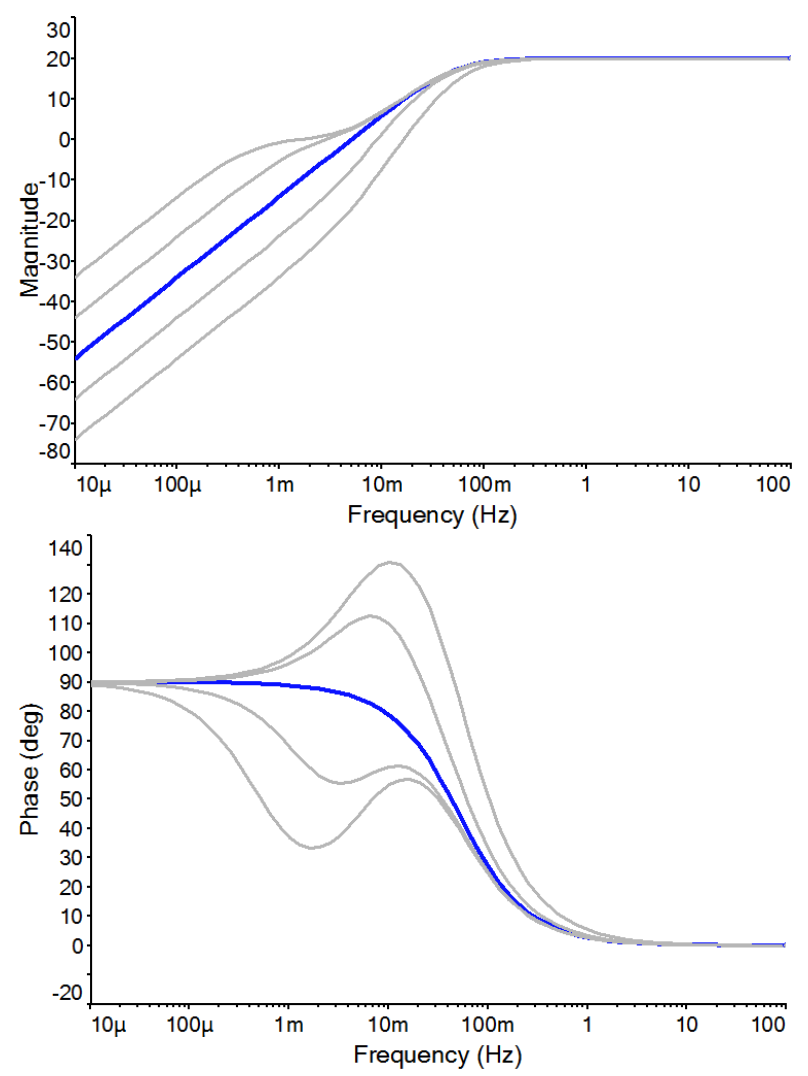

Figure 7. Magnitude and phase responses of 2-stage amplifier

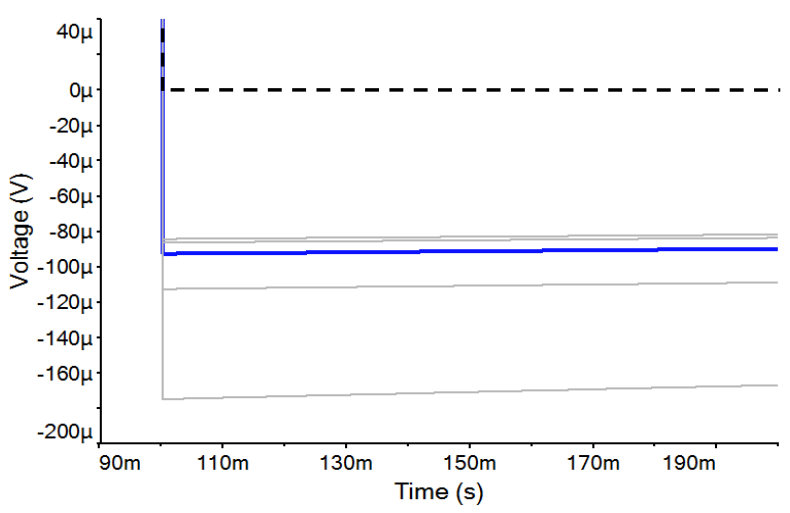

Figure 8. Trailing edge pulse response of 2-stage amplifier

\subsection{Three-stage differential amplifier}

The final configuration of interest is the 3-stage differential amplifier shown in Figure 9. This has a highpass input stage and a differential-to-single-ended output stage identical to the two stage amplifier. There is a second differential stage which is dc coupled to the first so that the output dc bias potentials of the first stage will carry through to the second stage. The ac coupling of the first stage is sufficient to block any dc polarisation potentials at the input. The steady state transfer function of this three stage amplifier is given as:

$$
\begin{aligned}
& \frac{V_{o}}{V_{1}-V_{2}}=\frac{R_{5}}{R_{4}} \times \\
& \frac{\left[1+j \omega C_{1}\left(R_{1}+2 R_{2}\right)\right]\left[1+j \omega C_{7}\left(R_{7}+2 R_{6}\right)\right] j \omega C_{3} R_{3}}{\left(1+j \omega C_{1} R_{1}\right)\left(1+j \omega C_{7} R_{7}\right)\left(1+j \omega C_{3} R_{3}\right)}
\end{aligned}
$$

where, $\mathrm{R}_{6}, \mathrm{R}_{7}$ and $\mathrm{C}_{7}$ are the components in the second differential stage. It can be seen that the dc coupling in the second differential stage adds an additional pole and zero to the transfer function. If the output stage has unity gain and the overall gain is shared equally between the two differential stages then the poles and zeros of both of these stages are identical so that $\omega_{P 1}=1 /\left(C_{l} R_{l}\right)=\omega_{P 3}=$ $1 /\left(C_{7} R_{7}\right)$ and $\omega_{Z 1}=1 /\left[C_{1}\left(R_{1}+2 R_{2}\right)\right]=\omega_{P I} / A_{V 1}=\omega_{Z 2}=$ $1 /\left[C_{7}\left(R_{7}+2 R_{6}\right)\right]=\omega_{P 3} / A_{V 2}$. The gains of the individual differential stages were kept at $20 \mathrm{~dB}$ as for the two-stage amplifier so that the overall gain of the three-stage amplifier is $40 \mathrm{~dB}$. On the basis of the results of Figures 7 and 8 for the two-stage amplifier the pole-zero cancellation was maintained in the first differential stage by maintaining the choice $C_{3} R_{3}=C_{l}\left(R_{1}+2 R_{2}\right)$. If the intention, in the first instance, is to maintain the $-3 \mathrm{~dB}$ frequency of the three-stage amplifier at the same location as for the two-stage amplifier then it can be shown that:

$$
\omega_{-3 d B 2}=\sqrt{\sqrt{2}-1} \omega_{-3 d B 1}=0.645 \omega_{-3 d B 1}
$$

where $\omega_{-3 d B 2}$ is the $-3 \mathrm{~dB}$ frequency of a single stage in a two-stage amplifier having identical stages and $\omega_{-3 d B I}$ is the $-3 \mathrm{~dB}$ frequency of a single-stage amplifier which is equal to the overall $-3 \mathrm{~dB}$ frequency of the two-stage amplifier. If $f_{-3 d B 1}=0.05 \mathrm{~Hz}$ then $f_{-3 d B 2}=0.032 \mathrm{~Hz}$ to maintain an overall $-3 \mathrm{~dB}$ frequency of $0.05 \mathrm{~Hz}$ for the three-stage amplifier. However, this choice does not allow the three-stage amplifier to meet the pulse response requirements. In order to accomplish this, it is necessary to lower the $-3 \mathrm{~dB}$ frequency of the three-stage amplifier to $0.043 \mathrm{~Hz}$ which corresponds to a $-3 \mathrm{~dB}$ frequency for the individual differential stages of $0.028 \mathrm{~Hz}$. This gives an undershoot of $98 \mu \mathrm{V}$ and a recovery slope of $15 \mu \mathrm{Vs}^{-1}$. This can be seen in Figures 10 and 11 where the optimised response is represented by the bold curves. The magnitude and phase responses are shown in Figure 10, while the pulse response expanded in the region of the undershoot is shown in Figure 11. This heavy grey curves represents the corresponding responses of a single-stage amplifier with a $-3 \mathrm{~dB}$ frequency of $0.05 \mathrm{~Hz}$. 


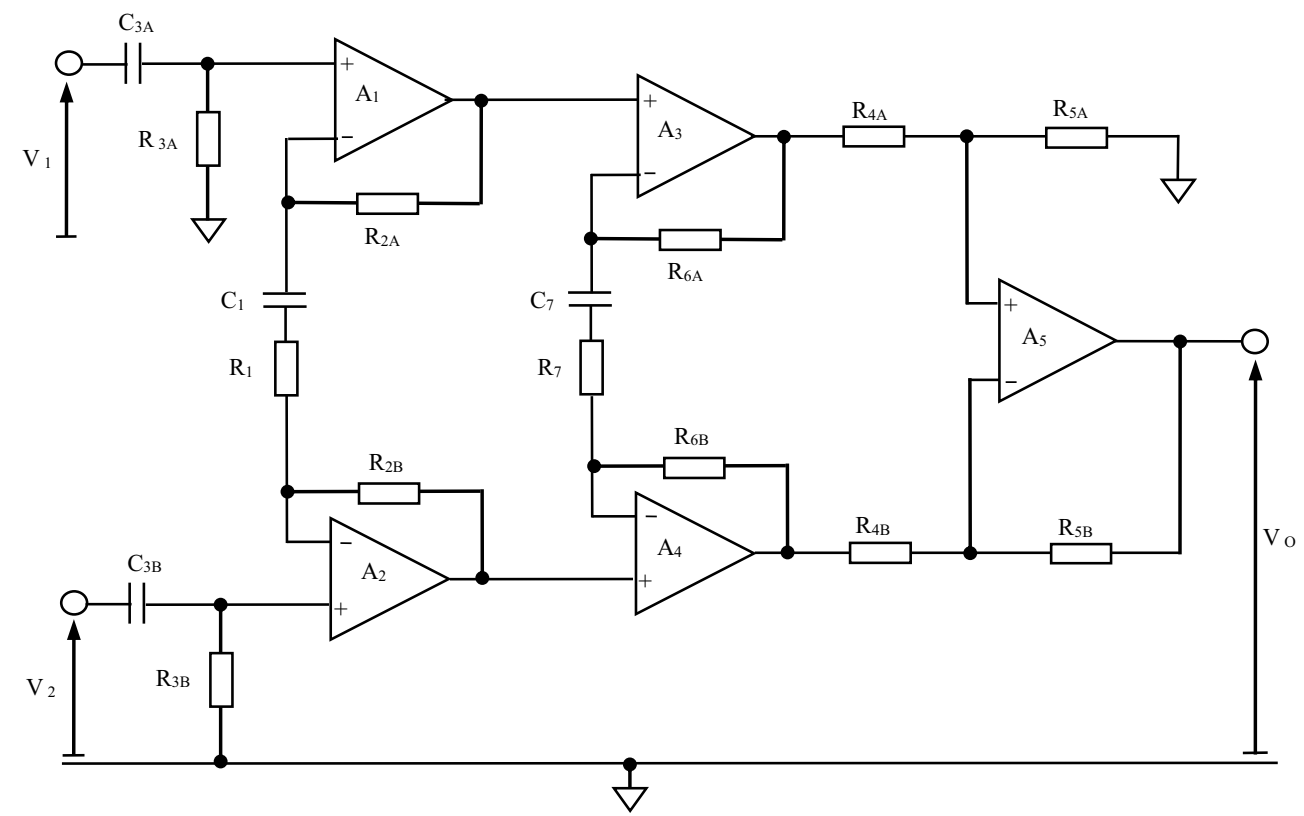

Figure 9 Schematic diagram of a 3-stage ECG amplifier
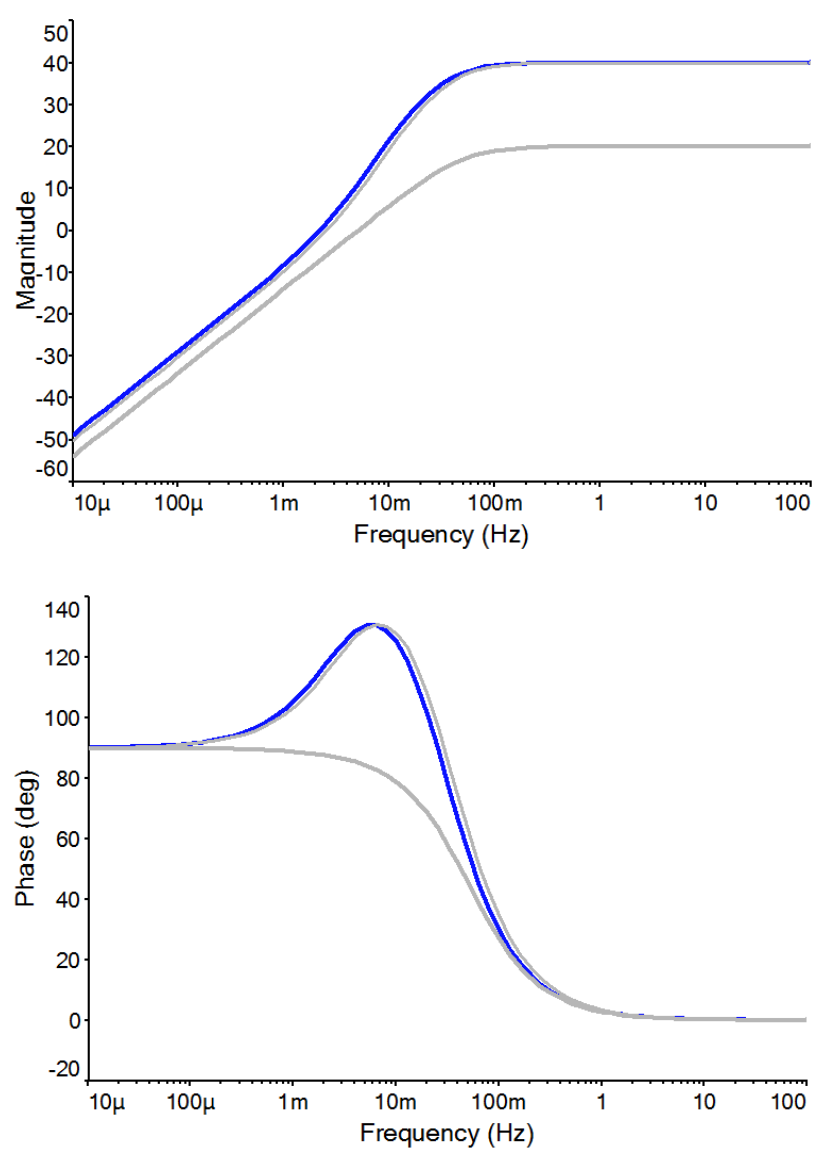

Figure 10 Magnitude and phase responses of 3-stage amplifier

\section{Conclusion}

A 3-stage ECG amplifier for use with unjelled electrodes has been designed which satisfies the IEC 60601 specification. The undershoot in the pulse response is less than $100 \mu \mathrm{V}$ and the recovery slope less than $300 \mu \mathrm{Vs}^{-1}$. The $-3 \mathrm{~dB}$ low cut-off frequency of two identical cascaded differential stages must be set at $0.028 \mathrm{~Hz}$ in order to accomplish this.

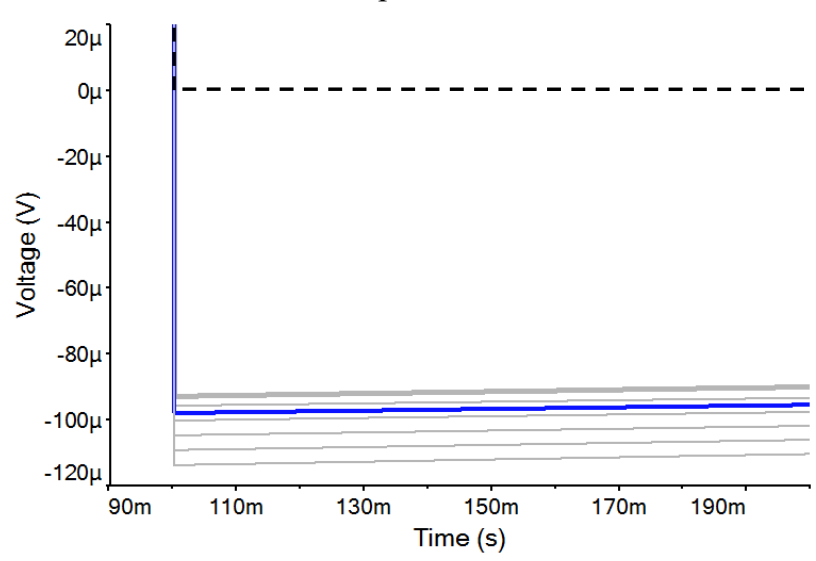

Figure 11 Pulse response of the 3-stage amplifier

\section{References}

1. International Electrotechnical Commission, Medical Electrical Equipment Std. 2-25. IEC 60601, 2011.

2. International Electrotechnical Commission, Medical Electrical Equipment Std. 2-47. IEC 60601, 2012.

3. A. S. Berson and H. V. Pipberger, 'The LowFrequency Response of Electrocardiographs, A Frequent Source of Recording Errors'; Amer. Heart J.; 71, 779-789, 1966.

4. D. Tayler and R. Vincent, 'Signal distortion in the electrocardiogram due to inadequate phase response', IEEE Trans. Biomed. Eng.; 30, 352-356, 1983.

5. C. E. Kossmann et al., 'Recommendations for standardization of leads and of specifications for instruments in electrocardiograpy and vectorcardiography.; Circulation, 35, 583-602, 1967.

6. H. V. Pipberger et al., 'Recommendations for standardization of instruments in electrocardiography and vectorcardiograhy, IEEE Trans Biomed. Eng.; 14, 60-68, 1967. 